\title{
Tangence
}

\section{Dramaturgies et refus de l'écrivain en Ontario français}

\section{François Paré}

Numéro 56, décembre 1997

Postures scripturaires dans la littérature franco-ontarienne

URI : https://id.erudit.org/iderudit/025958ar

DOI : https://doi.org/10.7202/025958ar

Aller au sommaire du numéro

Éditeur(s)

Tangence

ISSN

0226-9554 (imprimé)

1710-0305 (numérique)

Découvrir la revue

Citer cet article

Paré, F. (1997). Dramaturgies et refus de l'écrivain en Ontario français.

Tangence, (56), 66-79. https://doi.org/10.7202/025958ar d'utilisation que vous pouvez consulter en ligne.

https://apropos.erudit.org/fr/usagers/politique-dutilisation/ 


\section{Dramaturgies et refus de l'écrivain en Ontario français}

François Paré

Et puis? Ce n'est que du papier. L'histoire, la vraie, elle est dans la chair. In the blood and in the bones.

Le Colonel ${ }^{1}$

\section{Le spectacle et la mort}

Dans un article de la revue Liberté en 1995, Alain Roy plaçait sous l'égide menaçante du dieu de la mort, Thanatos, toute la génération des années 90 , dont le projet est aujourd'hui, disait-il, de * contourner* l'illusion du plaisir (l'érotisme généralisé: la consommation) léguée par la génération antérieure ${ }^{2}$. Or, dans la société nord-américaine, le refus du plaisir de consommer n'entraîne-t-il pas inévitablement le déni de l'être? Qui suis-je, hors de cette appropriation passagère du plaisir? La conscience aiguë de la prédétermination du spectacle, jusque dans sa réalisation ultime à travers la violence et la mort, entraîne donc une profonde remise en question du sujet. Car comment peut-on - contourner - cela même qui définit les règles du jeu dans la société actuelle et qui, à travers ces règles, permet aux individus de se constituer en tant que sujets vivants? Et quel est le rôle du langage dans ce plaisir illusoire où le sujet se perd? Comment l'éviter, cet artifice porteur de l'illusion, comment faire éclater *les mots vibrant dans ce qui est déjà fixé en eux. ${ }^{3}$ ?

Cette représentation d'un sujet, matériel et spirituel, toujours nourri de sa propre perte, faisant éclater le langage qui le constitue, force l'écrivain non seulement à adopter une position de refus de la jouissance généralisée, mais à se replier dans des

1 Michel Ouellette, Corbeaux en exil, Hearst, Le Nordir, 1992, p. 13

2 Alain Roy, - Le refus total *, Liberté, $\mathrm{n}^{\circ} 221$, octobre 1995, p. 80-100.

3 Claude Beausoleil, "La mémoire du livre., dans $I l y$ a des nuits que nous babitons tous, Saint-Lambert/Pantin, Éditions du Noroît/Le Castor Astral, 1986, p. 105. 
modes autodestructeurs du sujet individuel. "La jeune génération, écrit Roy, hérite des pulsions de mort non reconnues de ses prédécesseurs et retourne contre elle-même cette agressivité qui n'est pas la sienne. (p. 93). L'analyse d'un certain retour du désespoir dans l'ensemble des cultures d'Occident a le mérite de situer le débat, non plus au plan des collectivités, mais au niveau plus fragile des individus eux-mêmes, entraînés dans des systèmes affolants d'appropriation et d'ascèse qu'ils sont désormais seuls à investir. Le fatalisme et la recherche du dénuement frappent donc partout, autant le spectacle dégradé de la matérialité quotidienne (la rue, la maison, la chambre, le parc, le bar, la ville, le pays) que celui de l'esprit (la pensée, la théorie, le savoir, le livre, le texte, le chant, la scène), où circulent, dans notre société, les figures éperdues de la solitude.

De tous les modes d'expression qui alimentent notre modernité propre, c'est le théâtre qui permet de poser le plus clairement la question des liens difficiles qui unissent la matérialité dụ quotidien et une certaine quête de la spiritualité, souvent associée paradoxalement à l'exercice de la voix. Or, nulle part ailleurs que dans le thêâtre, sous ses multiples formes objectales (le corps, la scène, la voix, la lumière), a-t-on pu observer avec autant de clarté, surtout dans le théâtre nord-américain, le glissement vers une problématique de l'ascèse et de la mort, dans laquelle s'offre en sacrifice, par son absence spectaculaire, la figure inaugurale, entre autres, de l'écrivain. Car l'écrivain, au sens général, ést désormais investi des pulsions de la mort, celles qui annoncent depuis toujours sa disparition (violente) en tant que sujet autoritaire: son appauvrissement progressif, puis l'horizon de son absence catastrophique dans le spectacle qui sans cesse s'annonce. Cela se passera toujours sans lui: la représentation théâtrale lui sera funeste.

Le thêâtre franco-ontarien, dont il est question ici, est traversé depuis 30 ans par de telles pulsions de mort et par de tels schèmes autodestructeurs de l'identité subjective. En cela, ce thêâtre, souvent le produit de créations collectives, est parfaitement exemplaire. Son succès actuel et surtout l'impact relatif de certains de ses concepteurs les plus acharnés sur l'ensemble du théâtre francophone en Amérique témoigne de la modernité d'une sensibilité à la mort et au pouvoir de l'écriture qui découlait, au départ, du simple vécu de la culture minoritaire. Au premier plan, le théâtre franco-ontarien a posé la question de la 
68

nécessité même de l'écrivain, symbole de l'excédentaire dans une culture "pauvre", vivant avant la lettre le dénuement et le refus.

D'un côté, il arrive parfois que cette figure inaugurale de l'écrivain - Père, Mère, Récit -, telle que la produit le jeu théâtral, fasse l'objet d'une recherche désespérée, comme c'est sans doute le cas dans bon nombre de pièces franco-ontariennes, des premières œuvres d'André Paiement au Chien de Jean Marc Dalpé ou à French Town de Michel Ouellette. Mais il se peut aussi, comme dans les cuvres monologiques les plus récentes de Robert Marinier (L'insomnie, notamment), que le texte se renverse entièrement vers la matérialité de l'acteur, seul sur scène, seul responsable de l'enclenchement et du dénouement de la représentation, de sa représentation. Alors, dans ce cas, la figure inaugurale s'estompe, pour laisser place à une espèce de plaisir lié à la matérialité éphémère de la voix, celle du récit proprement récité.

\section{Mort de la figure écrivante}

Tes un parasite. Tu vis sur les rêves des autres. Tu fais du cash sur nos désirs.

Comment veux-tu que je te croies! On peut jamais savoir la couleur de ce qui sort de ta bouche.

Betty $^{4}$

C'est donc dans le contexte d'un puissant discours sur la mort, dont l'article d'Alain Roy faisait état, que doit se placer ici, à mon sens, une réflexion générale sur le rôle de l'écrivain en Ontario français. Le théâtre minoritaire, tel qu'il s'est constitué en Ontario français depuis 30 ans, a posé de manière remarquable la question des liens entre le sujet identitaire et le pouvoir, et, audelà de cette question, la nécessité même de la figure écrivante, toujours apparemment liée, elle aussi, aux structures de l'oppression.

En Ontario français, la question de l'identité collective s'est révélée centrale à tous les débats depuis le tournant des années

4 Michel Ouellette, Le hateleur, Ottawa, Le Nordir, 1995, p. 58. 
70. La description que fait de cette préoccupation Linda Cardinal est en tous points exemplaire:

Ainsi, l'identité franco-ontarienne devient, pendant les années 1960-1970, une idée-force, un principe rassembleur en vue de transformer la situation de domination du groupe, notamment à travers ses luttes pour les écoles françaises et le contrôle de ses institutions. [...] Artistes, littéraires et féministes se rallient à ce mouvement qui veut rapatrier le procès de construction de l'identité. 5

Ce récit fondateur de la communauté, nous le verrons, est apparu à la fois nécessaire et douloureusement problématique. En effet, dans toute communauté à la recherche de son identité propre, l'intervention de l'écrivain sur le récit fondateur est vouée à l'éphémère, puisqu'elle est toujours trop fabriquée, trop contrainte, trop chatoyante. L'écrivain s'institue par son langage métamorphique comme la condition du récit identitaire et pourtant son plus grand obstacle. Ce récit, repris par la coutume, devient alors le plus souvent l'évocation de la mise en accusation de l'écrivain et éventuellement celle de son retrait stratégique.

Pour la dramaturgie franco-ontarienne, la notion d'écrivain est donc apparue très tardivement, comme un fait d'institution plutôt que comme une réalité structurante. Le dramaturge y est devenu peu à peu un enchanteur public, lui-même acteur et spectateur: il n'a nullement à écrire. Son action sur le monde se passe justement de l'écriture. L'œuvre dramatique, si elle doit être métaphoriquement le produit de l'ontogenèse de la communauté, peut sans doute se passer d'une singularité jugée prétentieuse et blessante. Alors qu'on veut coûte que coûte favoriser l'échange et le foisonnement, l'écrivain semble faire appel aux images de fixité et de mort. L'ensemble du théâtre franco-ontarien, à partir du tournant des années 70 , a voulu mettre un terme à sa singularité, à la singularité qui menaçait - du moins est-ce ainsi que l'écrivain se laissait concevoir alors - tout l'imaginaire de la communauté ${ }^{6}$. Dans un tel programme, l'écrivain ne pouvait plus

5 Linda Cardinal, - Ruptures et fragmentations de l'identité francophone en milieu minoritaire; un bilan critique ", saciologie et sociétés, vol. XXVI, $\mathrm{n}^{\circ} 1$, printemps 1994, p. 75.

6 - Ia véritable singularité, écrit Stéphane Lépine, n’a jamais eu, contrairement aux standards programmés, la vertu de rassembler, de faire communier le plus grand nombre. [...] [L]e public sait confusément que chaque chose ou 
70

vraiment imposer l'hégémonie de son regard incontesté sur le langage.

Dans la culture opprimée - et c'est bien une telle conscience de l'oppression qui anime l'Ontario français depuis 30 ans! -, l'écrivain, pur avatar de la conscience, peut-il encore réclamer sa place initiale au sein d'une prise de parole qui, immanquablement, se tourne contre toutes les figures honnies de l'autorité? Trop transitive, la figure écrivante s'efface devant la circularité du rituel unissant acteurs et spectateurs dans un pur éloge de la parole. Cet effacement - et je me fais ici l'écho des merveilleux textes de Victor-Lévy Beaulieu sur le théâtre - appartient spécifiquement aux communautés opprimées :

lorsqu'on est Québécois, l'on risque, plus que quiconque, de passer sa vie à ne faire que ça, dérisoirement, grotesquement - ces entrées et ces sorties de scène qui témoignent fort bien de notre situation historique qui, pour paraître ambiguë relève du monde théâtral : [...] n'étant rien et satisfaits de l'être, nous n'avions aucun intérêt à entrer dans le grand cirque des nations, notre matérialisme a-historique permutant toutes les données, faisant de nous des missionnaires mais curieusement sans mission parce que n'étant d'aucun pouvoir, sinon celui de la Parole. ${ }^{7}$

Appartenir à la :Parole •! En effet, voilà qui était bien le projet du théâtre en Ontario français, éloignant du même coup la communauté des acteurs-créateurs de la figure écrivante, oppressive et moqueuse. Les premières ouvres montées après 1973 environ par le Théâtre du Nouvel-Ontario (T.N.O.) à Sudbury, puis ensuite notamment par le Théâtre de la Corvée à Ottawa, s'offraient comme de grandes fresques de création collective. La voix auctoriale y était elle-même plurielle, du moins officiellement.

Ce parti pris pour la création collective a découlé, en 1973, non seulement de critères esthétiques qui appartenaient à l'œuvre, mais de l'implication politique du jeu théâtral dans une

chaque corps réellement singulier qui apparaît sur une scène nous renvoie à notre unicité, c'est-à-dire à notre solitude et à notre différence irréductible en face de lui, autant dire à l'impossible achèvement du programme et à la mort. - Stéphane Lépine, "Seul ou avec d'autres: 50 ", Cabiers de théâtre Jeu, no 75,1995 , p. 85.

7 Victor-Lévy Beaulieu, • Le théâtre de la folie ", dans Entre la sainteté et le terrorisme. Essais, Montréal, VLB éditeur, 1984, p. 406-407. 
communauté coupée de l'écriture. Dans une telle communauté démunie, ne fallait-il pas que l'écrivain n'écrive pas, qu'il sache se placer, comme dans son essence même, du côté du jeu corporel du comédien et du spectateur, en dehors de l'écriture, dans une prise directe avec la réalité? Ne fallait-il pas que, dans une société dé-réalisée, le théâtre, intervention première sur la conscience collective, se tourne vers le donné réel? C'était là, dans un combat hautement ritualisé, que la question de la survie se posait, $a ̀$ l'exclusion même de l'écrivain. Dans le choix entre la *Parole * et la "Loi , il est clair que la dramaturgie franco-ontarienne moderne a privilégié pour un temps la .Parole *, en vertu de son incandescence publique, quitte à composer avec sa faiblesse institutionnelle. Parler d'abord, écrire ensuite. Un tel choix supposait la rature de l'écrivain ou au moins la mise en scène de son absence dans des "textes" qui, objets de continuels remaniements scénographiques, résisteraient à toute fixité.

Il faut dire que le théâtre franco-ontarien s'est inscrit dès le tournant des années 70 dans une volonté de redéfinition de la scénographie qui traversait et continue de traverser le continent nord-américain dans son ensemble. Dans ce sens, l'Ontario français a été très à l'écoute des théories de la "performance *, celles de Richard Schechner, par exemple, et s'est posé dans le contexte bien particulier de la culture minoritaire la question de la relation problématique entre le dramaturge, le support textuel, le jeu théâtral et le spectateur ${ }^{8}$. Dans ce déplacement du théâtre vers un espace du jeu, conçu comme lieu de libération individuelle et collective, l'écrivain s'est trouvé marqué par sa position d'extériorité, puisqu'il semblait évoluer hors des "frontières * de l'événement théâtral, qu'il ne pouvait que par substitution prendre part à ce jeu libérateur dont il n'était parfois que l'élément déclencheur et parasitique. Cet effacement de l'écrivain-dramaturge ne se produisait pas uniquement au moment d'effectuer les choix scénographiques et de concevoir un : script *: il était constitutif de l'efficacité de l'action thêâtrale sur la communauté des sṕectateurs; il était au cœur de la fusion entre le matériel et le spirituel.

8 Voir Richard Schechner, The Future of Ritual, London/New York, Routledge, 1993. Schechner rappelle que le thêâtre est, dans le sens où il l'entend, un - entraînement au rêve. (dream-training). La mise en scène du rêve (inclividuel, communautaire) entraîne une étrange fusion entre la virtualité et la matérialité de l'imaginaire, une sorte de corporalité du spirituel (Chapitre 7 , *The Future of Ritual , p. 228-265). 
72

\section{Les ruses traductives}

Y t'aiment, icitte. Ben contents de t'avoir, eux autres. $Y$ s'imaginent que t'es assez intelligent pour les sortir du trou. S'imaginent que tu vas les sauver. Vont t'élire maire de la place aux prochaines élections, sûre de ça... Mais ... Ah, pis fuck!

Cindy"

Certes, le théâtre franco-ontarien, peut-être d'abord de manière intuitive, a voulu rompre avec l'univocité de l'emprise idéologique. Il s'est orienté assez rapidement vers une production théâtrale à ras de terre, une "ruse traductive, une reddition stratégique •, pour reprendre les termes d'Obed Nkunzimana parlant du roman africain ${ }^{10}$, s'efforçant d'abolir l'écart entre la dramaturgie et l'espace de sa réception. Il était facile de conclure, comme l'a fait en réalité l'ensemble du théâtre de revendication en Amérique du Nord, que, de tous les aspects qu'il fallait soumettre à l'expérience du soupçon, c'était celui du texte produit par un écrivain qu'on se devait avant tout de contester.

En effet, comment nier que dans cette société même c'était le "texte" qui faisait écran, que ce texte", défini par sa fixité et par son origine dans la singularité de son auteur, ne pouvait être, dans les mots de Kantor, qu'-un corps étranger ;, dans ses rapports à la fois au jeu théâtral et à la société minoritaire dans son ensemble? ${ }^{11}$

Inversement, le théâtre franco-ontarien, dans les créations collectives du Théâtre de la Corvée ou de Vox Théâtre, par exemple, continuait d'affirmer la ritualisation propre au jeu théâtral. À l'événement du théâtre joué, on attribuait volontiers les capacités rédemptrices, chamaniques, du rituel, comme si l'évanescence de l'acte pouvait en évacuer toute la charge idéologique, tout le

9 Michel Ouellette, French Toun, Ottawa, Le Nordir, 1994, p. 72.

10 Obed Nkunzimana, "Les stratégies postcoloniales et le roman francophone: débat théorique et prospective critique ., Présence francopbone, $n^{\circ} 50,1997$, p. 7-26.

11 Le théâtre, disait Kantor dans un manifeste de 1966, n'est pas un appareil à reproduire la littérature. Au contraire, il fallait enclencher un *processus de désillusion. que Kantor associait à la critique de la figure dominante de l'écrivain. Voir Tadeusz Kantor, Le théâtre de la mort, textes réunis par Denis Bablet, Lausanne, L'Âge d'homme, 1977, p. 67 suiv. 
caractère autoritaire. Ainsi, les premières créations collectives présentées dans la région de Sudbury au début des années 70 (Lavalléville d'André Paiement ou Les communords de Claude Belcourt) faisaient vivement appel au caractère chamanique de la représentation théâtrale, à ses conditions cérémoniales. "Le plus pressant, écrivait Normand Renaud dans un article sur le Théâtre du Nouvel-Ontario en 1992, sera toujours de révéler une communauté à elle-même, de l'assurer de sa raison d'être et d'inventer ses avenirs possibles, envers et contre le constat quotidien de sa précarité ${ }^{12}$. Ce programme, commencé au tournant des années 70 , trouvait donc encore très récemment un appui indéfectible. Pourtant, la quête de la révélation identitaire suppose toujours le renversement de la figure écrivante. Il se peut que la "séparation par laquelle la parole parle - soit aussi vécue, projetée dans la cérémonie scénique comme une douloureuse vacuité. Le théâtre ne donne donc pas "congé à la présence", comme le pensait Claude Lévesque, mais il est plutôt rempli de la apensée magique. sur laquelle les rapports entre les individus peuvent être rêvés autrement, dans une fusion du dehors et du dedans ${ }^{13}$. Il n'est sans doute pas étonnant que, dans les conditions de dissociation et d'invisibilité dans lesquelles évoluent les sociétés opprimées, la puissance "magique " du jeu théâtral acquière une importance presque insoutenable.

En outre, il est sûr que la faiblesse institutionnelle, caractéristique d'une culture minoritaire comme celle de l'Ontario français, a contribué largement à la dissolution de la figure monolithique de l'écrivain. Au théâtre, la pluridisciplinarité est aujourd'hui de mise. Pour des raisons économiques autant qu'idéologiques, il arrive donc le plus souvent que l'écrivain ne soit jamais bien longtemps écrivain à part entière et qu'il se métamorphose à son tour en comédien et metteur en scène de sa propre pièce : Robert Marinier interprétant Robert Marinier, Robert Bellefeuille interprétant Robert Bellefeuille, Jean Marc Dalpé interprétant Jean Marc Dalpé. Et ainsi de suite. Si Carlos Fuentes, dramaturge mexicain, disait ne pouvoir inventer ses personnages féminins autrement que joués par les plus grandes actrices, Máría Félix et Dolores del

12 Normand Renaud, "le charisme du Théâtre du Nouvel-Ontario : la griffe Sudbury , Liaison, $n^{\circ} 69$, novembre 1992, p. 24.

13 Voir l'article toujours pertinent de Claude Lévesque, : le temps hors temps de l'écriture *, Brèches, $\mathrm{n}^{\text {os }}$ 4-5, printemps-été 1975, p. 94-118. 
Río ${ }^{14}$, nombre de dramaturges franco-ontariens n'ont pu imaginer leur "écriture "que dans le corps de leur propre personnage. En fait, n'est-il pas possible de voir, à travers la polyvalence forcée qui est le lot des "pauvres", une des conditions mêmes de la "Parole", de sa matérialité multiple? Là, en effet, peuvent surgir les conditions de renversement de l'autorité scripturale; là l'écrivain disparaît pour renaître autrement, dans un autre corps, libre de jeu et de parole, à la fois lui-même et autre.

Partout dans le théâtre de création de la fin des années 70 en Ontario français, l'écrivain s'est donné à vivre le fantasme ultime de sa transformation en acteur, de son incarnation lumineuse devant un auditoire convié à chaque fois à l' entrée en matière " de la figure écrivante, et puis éventuellement à sa disparition ${ }^{15}$.

\section{«C'est moi l'auteur": Michel Ouellette}

Ma rage est vieille de cing cents ans.

Virgile

Toé, t'es rien que la peur dans le ventre. Toé, m'as t'écraser. Pas besoin d'argent sur la table. Pas besoin de gageures. Toé, m’as te défaire, juste pour le plaisir.

Dempsey ${ }^{16}$

Pour donner corps à ces quelques réflexions, je me propose maintenant de *relire", très brièvement, trois œuvres du dramaturge Michel Ouellette, dont les propos en exergue m'ont accompagné jusqu'à maintenant. Car plus que tout autre, dans la dramaturgie récente en Ontario français, Ouellette résume la descente aux enfers de l'écrivain, incapable d'imposer aux multiples voix du présent la singularité de son récit. Ce sera d'abord le texte - puisqu'il s'agit d'un texte - de Corbeaux en exil, présenté en lecture publique en

14 Voir le prologuc de la pièce Orquideas a la luz de la luna de Carlos Fuentes, Barcelone, Seix Barral, 1982, p. 9.

15 Voir Michel Corvin, "la créativité dite métaphore., dans Emmanuel Jacquart (dir.), Theâtre et création, Paris, Champion, 1994, p. 33-46: * S'agissant du théâtre, le symbole y prend (ou y retrouve) toute sa valeur matérielle, concrète, d'objet - (p. 36).

16 Michel Oucllette, Le bateleur, op. cit., p. 87, 119. 
mars 1991, puis publié l'année suivante; dans cette ouvre moins connue, le dialogue s'ouvre justement sur l'entrée en scène de l'écrivain (Pete: "C'est moi l'auteur"). Elle marque une période où Ouellette s'interroge ouvertement sur le rôle de l'écrivain dans la construction de l'" histoire " individuelle et collective.

Ensuite, je voudrais proposer une brève analyse de Frencb Town, œuvre moins appréciée de la critique franco-ontarienne, où se trouve posée pourtant implicitement la question du rôle dialogique attribué à l'écrivain, désormais hors-scène. Enfin, Le bateleur, présentée à Sudbury en 1995 et publiée la même année, pose la question des schèmes autodestructeurs qui sont à l'origine de la communauté opprimée et des individus qui la composent. Ces œuvres de Michel Ouellette sont exemplaires. Celui-ci, ni acteur, ni metteur-en-scène, est sans doute le "premier " véritable écrivain de théâtre de l'Ontario français ${ }^{17}$.

Au premier regard, c'est dans Corbeaux en exil que la figure de l'écrivain se trouve inscrite dans toute sa prégnance. Ici règnent les lieux clos, les infirmités et la mort. Nulle identité, nulle vie sans qu'y soit inscrit le passage fatidique des corbeaux. La pérennité de ces oiseaux rapaces, grands survivants de la lutte pour la vie, fait écho à la précarité des institutions et à l'instabilité des identités subjectives.

Le dialogue s'ouvre sur Pete, personnage central, auteur d'un futur roman devant raconter l'expérience des camps de prisonniers durant la Première Guerre mondiale à Kapuskasing. Corbeaux en exil est son histoire et ultimement celle de ses ancêtres, dont il retrace la part jouée dans l'établissement et le maintien des camps. Au départ, "tout est bien documenté * et le récit construit par l'auteur s'inspire entièrement du compte rendu de Watson Kirkconnell (récit réellement publié) sur les camps de prisonniers dans le Nord de l'Ontario. Cette référence à un document historique sert d'amarrage à toutes les divagations de Pete. C'est par ce document qu'il entend redécouvrir son passé morbide sans sombrer dans la folie, sans se soumettre aux puissances destructrices qui animent de tout temps la communauté.

17 Jean Marc Dalpé, Brigitte Haentjens, André Paiement, Robeıt Marinier, Robert Bellefeuille, Pier Roclier, Marie-Thé Morin, entre autres, sont tous à la fois dramaturges et comédiens-metteurs en scène. Confusion idéologique des rôles assignés, bien sûr : tout cela était souhaité. Chez Michel Ouellette, l'écrivain paraît joucr, à première vue, un rôle plus traditionnel, celui du simple producteur de l'apparcil textuel sur lequel se construira avec d'autres le jeu. 
76

L'auteur mis en scène par Michel Ouellette a d'abord tout pour réussir dans son entreprise de dévoilement du passé: il dispose de documentation abondante; sa mère et son entourage immédiat ne s'opposent pas à ses projets d'écriture:

Ah, je suis tellement contente. J'ai toujours voulu que tu deviennes un écrivain. Je le savais qu'un jour tu te déciderais (Corbeaux en exil, p. 18-19).

Pete dispose encore d'une capacité de transgresser les frontières temporelles et spatiales. Si le passé recèle des gestes peu courageux et des compromis douteux, sa découverte s'impose cependant avec force pour chacun des personnages de Corbeaux en exil, qui font appel à une certaine dimension onirique des répliques, s'enchaînant les unes les autres, comme un système d'échos et de similitudes. La recherche du passé ne mène guère ici à des découvertes irréconciliables; au contraire, malgré les images troublantes qu'il suscite, le passé, en tant que récit, sert à former les bases d'une communauté du partage, d'une communauté fondée sur les structures de l'enfermement.

L'écrivain est donc très présent, essentiel même au déroulement de chacune des scènes. S'il intervient, c'est en tant que personnage, cependant. Les didascalies, réduites au minimum, ne font que marquer les temps d'arrêt. Pourtant, et c'est là ce qui m'intéresse ici, sa présence dans le corps de la pièce est très problématique. Aux premières heures, tout va bien. Encouragé par sa mère et par son milieu, Pete produit livre sur livre. Il ne remet pas en question sa quête d'une vérité documentée, représentée par le récit historique de Kirkconnell.

C'est au moment où survient la crise identitaire, le désir de "vivre en français" que Pete remet lui-même en question la nécessité de la figure écrivante et la véridicité de son entreprise: “Toute cette histoire est un grand mensonge que je raconte à ma mère parce que je suis pas capable d'avouer la vérité. (p. 27). Reconnaissant son impuissance, Pete joue peu à peu, à partir de la Séquence 1, un rôle effacé. Il coupe progressivement ses attaches avec sa mère et avec le désir d'être écrivain au sens où elle l'entend.

Alors qu'elle insiste pour que Pete ait le courage de raconter l'histoire familiale et les liens sordides qui lient sa famille aux camps d'incarcération de Kapuskasing, celui-ci répond qu'il n'a aucun talent: "Maman, je ne peux pas écrire notre histoire. Je ne la comprends pas. Elle m'échappe... Je préfère me taire ou ben tra- 
duire... Oui, je suis juste bon pour traduire. (p. 62). Pete perd alors tout rôle dans la suite des événements : il ne sera que l'observateur de dialogues qui lui échappent totalement et dont il ne peut assurer la véridicité. C'est que l'écrivain (Pete Tremblay - de par son nom trouble, le Canadien Français par excellence!) ne s'appartient pas: il ne peut être que le traducteur de son propre récit, incapable, en réalité, de produire de l'inédit dans le champ d'une réalité toujours inexplicablement honteuse. La démobilisation de l'écrivain à la fin de Corbeaux en exil révèle l'impossibilité de sa présence hégémonique dans la constitution du récit fondateur.

À première vue, Frencb Town, pièce écrite deux ans après Corbeaux en exil, semble entièrement libérée de la fonction auctoriale. L'écrivain n'y est certes plus personnage. Les acteurs de ce drame familial bénéficient d'une autonomie que le théâtre de Ouellette n'avait guère permise auparavant. Il est vrai que le récit fondateur, celui de l'incendie de French Town, raconté par la mère ("Everybody has a mother", avait bien dit le Colonel dans Corbeaux en exil), a toutes les apparences d'une métaphore pour la figure absente de l'auteur. Mais c'est ce récit même qui finit par discréditer la véracité de la figure écrivante.

French Town présente à la lecture - et encore plus à la mise en scène - une curieuse fragmentation. La version publiée de la pièce s'offre, en effet, comme une suite de tableaux thématiques très brefs, portant chacun un titre: "Souvenirs, Confrontation, La chasse, Pool room, Bingo, Au nom du père ", etc.). Il est difficile d'imaginer que la pièce puisse avoir une quelconque cohérence sans la succession de ces sous-titres, indiquant le plus souvent les lieux du passé. Dans sa critique de la représentation de French Town, coproduction du Théâtre du Nouvel-Ontario et du Théâtre français du Centre National des Arts en 1993, Bondfield Marcoux regrettait l'absence de moments charnières qui auraient permis de comprendre la complexité de la prise de conscience suscitée par le retour au bercail de chacun des membres de la famille:

On se découvre en train de combler les scènes manquantes du drame, par des explications hâtives qui pour éclairantes qu'elles soient, nous éloignent du spectacle. L'action n'est plus sur scène mais dans nos têtes. ${ }^{18}$ 
Il semble, pourtant, que la fragmentation du tissu de French Town soit déterminée par l'abstention stratégique de l'écrivain.

En effet, et cela n'était pas aussi audible dans la mise en scène de la pièce, c'est bien l'écrivain qui, comme dans le salon du psychanalyste, provoque par l'interjection répétée et concertée des titres thématiques le flot des souvenirs. Ainsi en est-il, par exemple, des mots * La chasse - qui provoquent chez Pierre-Paul et Cindy l'écho nécessaire à la mise en œuvre du souvenir: "La chasse, la tabarnak de chasse. (p. 28). Ou encore, pour ne citer que ces deux exemples, les mots "Les chambres", lancés par ce "souffleur" qu'est devenu le dramaturge, engendrent une nouvelle vague de souvenirs chez les trois personnages: "Ma chambre, une ostie de prison; ma chambre, un véritable refuge; ma chambre, c'était la chambre de Pierre-Paul. (p. 26). Il n'y aurait pas de dialogue possible sans le souffle de celui qui, implicitement, précède toutes les interventions. Dans French Town, le silence agit comme symbole de la figure écrivante, donc inaudible - d'où la difficulté de mettre cette pièce en jeu. L'écrivain, caractérisé par son absence, joue un rôle décisif dans l'engendrement du jeu théâtral. En même temps, sa mise en retrait ne permet plus la cohérence idéologique attribuée au passé dans Corbeaux en exil, de sorte que le récit fondateur, habité par la honte et les pulsions de mort, ne peut jamais se constituer pleinement.

Dans Le bateleur, enfin, on chercherait en vain la figure de l'écrivain. Créée en 1995, cette pièce se passe dans le bar désert d'un hôtel du Nord. Comme ailleurs dans l'œuvre de Michel Ouellette, l'espace est tragiquement fermé; seul la traversée difficile du temps, la recherche du passé obsédant, peut sans doute - mais est-ce illusoire? - permettre son ouverture. Ici encore, pourtant, malgré l'absence de l'écrivain, malgré le sentiment confirmé que les personnages sont parfaitement seuls dans leur enfermement, la mise en accusation de l'écrivain est omniprésente. Pour Ouellette, le théâtre est le lieu ritualisé de la circularité; le jeu n'excède jamais le cadre étroit de cette circularité. C'est là sa condition primordiale. Et ce vortex, sur lequel la communauté joue sa survie échappe à la détermination de l'écriture, du texte.

À noter que Pierre Karch fait part des mêmes réserves dans son compte rendu publié dans Francophonies d'Amérique, $n^{\circ}$ 5, 1995, p. 91-92. 
Le bateleur importe parce que, cette fois, nous est posée la question du théâtre. Quel est donc son rôle dans la rupture de l'enfermement? Quel est l'enjeu de l'écrivain, lorsqu'il s'abandonne stratégiquement pour laisser place au dialogue, à la confrontation, à l'affleurement de la honte? Le bateleur, par l'intermédiaire de Dempsey, sans doute le personnage le plus polyvalent créé par Ouellette, soulève la question de la parole, en tant qu'agent de rupture historique. Est-il possible de rompre le récit fondateur, sans avoir d'autre instrument pour le faire que la Parole même qui nourrit ce récit?

Dempsey, figure certainement diabolique, est ici un personnage à part entière, mais sa fonction est méta-théâtrale : c'est lui qui construit, puis déconstruit les conditions de la déchéance. Il est, comme il le révèle lui-même à la fin de la pièce, le metteur en scène: celui de al'éternel combat, entre le bien et le mal. (p. 95). Il n'est pas écrivain. Par son intervention négative, Dempsey permet l'entrée en scène de Virgile qui fera de la circularité du passé autochtone une réponse à l'ultime déchéance des institutions. Il y a ici, dans ce personnage de Virgile - le nom même de l'écrivain, s'il en est un! - , un refus paradoxal de la singularité de la figure écrivante et son remplacement par l'anonymat rédempteur du récit mythique. Il n'y a d'écrivain que Virgile. Et, aux fausses "mères " qui avaient hanté jusque là l'œuvre dramatique de Ouellette, mères misérables et enfermantes, Le bateleur substitue le personnage féminin, Eliza, devenue Betty (dans le chatoiement des identités), porteur de la rupture, capable d'engendrer la singularité.

Le théâtre semble donc se construire tout seul: "De la magie tout ça", s'écrie Dempsey dans Le bateleur. C'est d'une part son attachement pour le rituel qui permet de nier la présence en lui d'une voix auctoriale singulière. Il n'est pas étonnant que, dans la défaite de la figure écrivante, l'œuvre de Michel Ouellette se tourne à la fin vers l'évocation du mythe. Mais il faut aller plus loin. Car, dans le théâtre franco-ontarien, il est clair que l'ouverture tant cherchée des lieux d'enfermement et la compréhension même de ce qui dans toute communauté rend l'enfermement possible passent par une ascèse qui suspend provisoirement la pertinence de la figure écrivante. L'écrivain reste. On se réconforte de sa présence supposée. Mais le jeu théâtral est une incarnation * in the blood and in the bones": il tire toute sa "magien des ruses textuelles qui nous amènent à croire en du pur visible. 